\title{
Lower Bounds of Decay Order of Eigenfunctions of Second-order Elliptic Operators
}

\author{
Dedicated to Professor S. Mizohata on his 60th birthday
}

By

\section{Jun UCHIYAMA*}

\section{§. Introduction}

Let real-valued function $v_{i j}(y) \quad(0 \leq i<j \leq N)$ defined on $\mathbb{R}^{3}$ satisfy

$$
\begin{array}{ll}
v_{i j}(y) \rightarrow 0 \text { as }|y| \rightarrow \infty \text { in } \mathbb{R}^{3} & (0 \leq i<j \leq N), \\
v_{i j}(y) \geq 0 \text { for } y \in \mathbb{R}^{3} & (1 \leq i<j \leq N), \\
v_{i j}(y) \in L_{l o c}^{2}\left(\mathbb{R}^{3}\right) & (0 \leq i<j \leq N),
\end{array}
$$

and let

$$
\begin{aligned}
& H=-\sum_{i=1}^{N} \Delta_{i}+\sum_{i=1}^{N} v_{0 i}\left(x^{i}\right)+\sum_{1 \leq i<j \leq N} v_{i j}\left(x^{i}-x^{j}\right), \\
& D(H)=H^{2}\left(\mathbb{R}^{3 N}\right), \\
& \Sigma=\inf \sigma_{e s s}(H),
\end{aligned}
$$

where $x^{i} \in \mathbb{R}^{3}$, and $\Delta_{i}$ is a Laplacian in $\mathbb{R}^{3}$ with respect to $x^{i}$. Then by easylike application of Agmon [1, Theorem 5.2, p. 85] we have the following: If $u(x)$ satisfies

$$
\begin{aligned}
& (H u)(x)=\lambda u(x) \text { in } \mathbb{R}^{3 N}, \\
& u \in D(H), \text { and } \lambda<\Sigma(\leq 0),
\end{aligned}
$$

then for any $\varepsilon>0$ there exists some constant $G>0$ such that for a. e. $x=\left(x^{1}, \ldots, x^{N}\right) \in \mathbb{R}^{3 N}$ we have

$$
|u(x)| \leq C e^{-(1-\varepsilon) \sqrt{\bar{\Sigma}-\lambda|x|}}
$$

In this paper by Theorem 1.5 and 1.7 given in $\S 1$ we have: If, besides above conditions, $v_{i j}(y)$ satisfies

Communicated by S. Matsuura, June 13, 1985.

* Kyoto Institute of Technology, Matsugasaki, Sakyoku, Kyoto 606, Japan. 


$$
\begin{array}{r}
v_{0 i}(y) \leq 0 \text { is a homogeneous function of degree }-\left(\gamma_{0}-\varepsilon_{i}\right) \\
(1 \leq i \leq N), \\
\begin{aligned}
v_{i j}(y) \geq 0 \text { is a homogeneous function of degree }-\left(\gamma_{0}+\varepsilon_{i j}\right) \\
(1 \leq i<j \leq N),
\end{aligned}
\end{array}
$$

where $\varepsilon_{i}, \varepsilon_{i j} \geq 0$ and $0<\gamma_{0} \leq 2$ (this case has been considered in Mochizuki-Uchiyama [8, p. 131 p. 133]), then, since

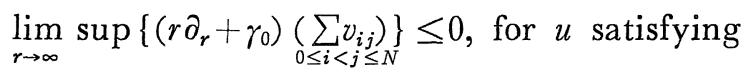

$$
\begin{aligned}
& -\sum_{i=1}^{N} \Delta_{i} u+\sum_{i=1}^{N} v_{0 i}\left(x^{i}\right) u+\sum_{1 \leq i<j \leq N} v_{i j}\left(x^{i}-x^{j}\right) u=\lambda u \text { in } \boldsymbol{R}^{3 N}, \\
& u \in H_{l o c}^{2}\left(\mathbb{R}^{3 N}\right)
\end{aligned}
$$

supp $[u]$ is not a compact set in $\mathbb{R}^{3 N}$,

$\lambda$ is a real constant,

we have for any $\varepsilon>0$

$$
\begin{array}{ll}
\lim _{R \rightarrow \infty} e^{2(1+\varepsilon) \sqrt{|\lambda|} R} \int_{R<|x|<R+1}|u|^{2} d x=\infty, & \text { if } \lambda<0, \\
\lim _{R \rightarrow \infty} e^{\varepsilon R} \int_{R<|x|<R+1}|u|^{2} d x=\infty, & \text { if } \lambda \geq 0 .
\end{array}
$$

If $v_{i j}$ is a Coulomb potential, namely,

$$
\begin{array}{ll}
v_{0 i}(y)=-\mathrm{Z}_{i}|y|^{-1} & (1 \leq i \leq N), \\
v_{i j}(y)=\mathrm{Z}_{i j}|y|^{-1} & (1 \leq i<j \leq N),
\end{array}
$$

where $Z_{i}, Z_{i j}$ are non-negative constants, $v_{i j}(0 \leq i<j \leq N)$ satisfies the all conditions as mentioned above.

In this paper we also consider general second-order elliptic equations as following:

$$
\begin{aligned}
& -\sum_{i, j=1}^{n}\left(\frac{\partial}{\partial x_{i}}+\sqrt{-1} b_{i}(x)\right) a_{i j}(x)\left(\frac{\partial}{\partial x_{j}}+\sqrt{-1} b_{j}(x)\right) u(x) \\
& +\left(q_{1}(x)+q_{2}(x)\right) u(x)=\lambda u(x)
\end{aligned}
$$

(in some neighborhood of infinity of $\boldsymbol{R}^{n}$ ).

Our main assumptions are the following:

$\operatorname{curl} b(x)=o\left(r^{-1}\right)$,

$q_{1}(x)=V_{1}(x)+V_{2}(x)$,

$V_{1}(x)$ satisfies the similar conditions as given for above $v_{i j}$,

$V_{2}(x)=o(1)$ and $\partial_{r} V_{2}(x)=o\left(r^{-1}\right)$

$q_{2}(x)=o\left(r^{-1 / 2}\right)$ 
at infinity. More detailed conditions are stated in $\S 1$. Then $u$ has a similar lower bound of decay order at infinity as given above.

Our results stated in $\S 1$ can be considered as a generalization of Bardos-Merigot [2, Theorem 2.2 (ii) and (iii), p. 329, which seems to need some modifications of statements of Theorem 2.2, and treats the case $\left.a_{i j}(x)=\delta_{i j}, b_{i}(x)=q_{2}(x)=V_{1}(x)=0\right]$. Our treatment of $b_{i}(x)$ can be found in Ikebe-Uchiyama [5], Kalf [6], Mochizuki [7] and Eastham-Kalf $[3, \S 6]$. Our method of proofs is a short-cut of Roze [9] and Éidus [4]. Lastly we remark that if we assume a stronger condition $q_{2}(x)=o\left(r^{-1}\right)$, then for $\lambda$ satisfying $\gamma_{0} \lambda>\lim \sup \left\{r \partial_{r} q_{1}(x)+\gamma_{0} q_{1}(x)\right\} \quad\left(0<\gamma_{0}<2\right)$ we have

$$
\lim _{R \rightarrow \infty} R^{\left(r_{0} / 2\right)-1+\varepsilon} \int_{R_{0}<|x|<R}|u|^{2} d x=\infty \quad \text { for any } \varepsilon>0 .
$$

(See, Uchiyama [10], Mochizuki [7] and Mochizuki-Uchiyama [8].)

\section{§1. Notations and Main Results}

At first we shall list the notations which will be used freely in the sequel:

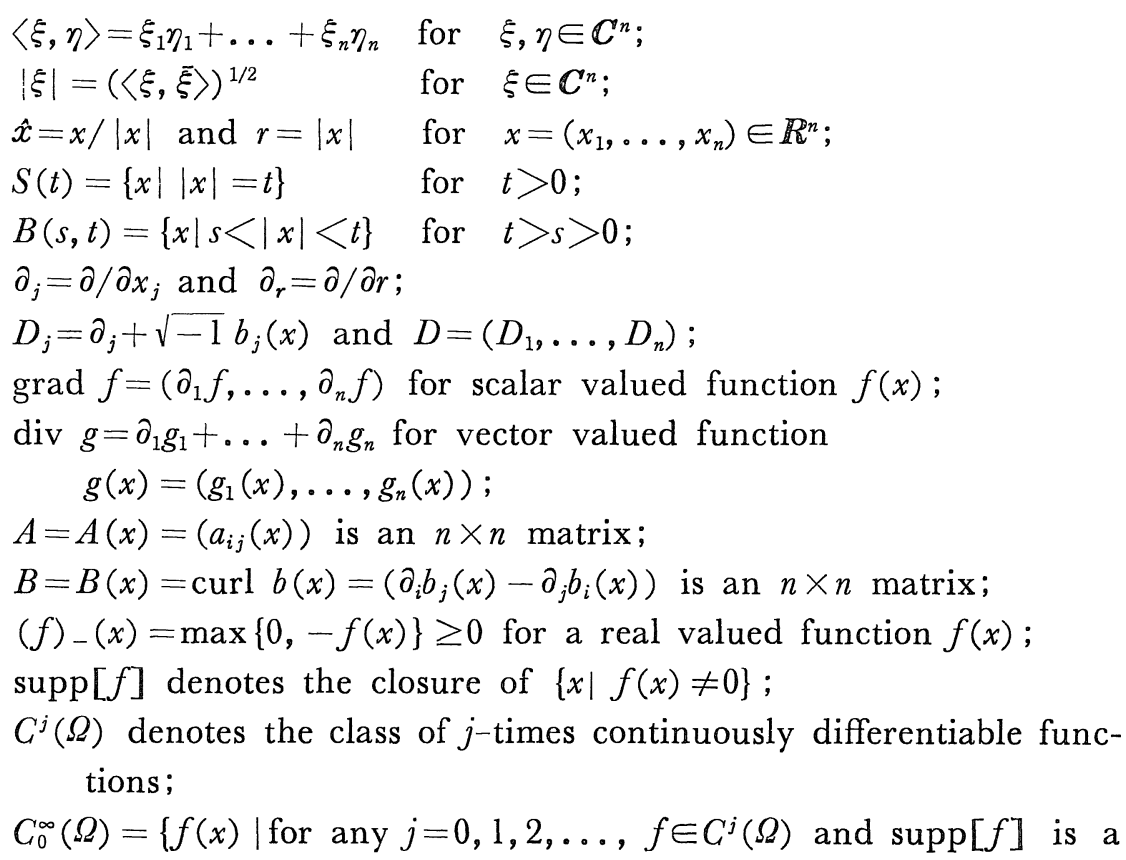


compact set in $\Omega$; ;

$L^{p}(\Omega)=\left\{\left.f(x)\left|\int_{\Omega}\right| f(x)\right|^{p} d x<\infty\right\}$ for $p \geq 1 ;$

$L_{l o c}^{p}(\Omega)=\left\{f(x) \mid\right.$ for any compact set $\left.K \subset \Omega, \int_{K}|f(x)|^{p} d x<\infty\right\}$ for $p \geq 1$;

$H^{m}(\Omega)$ denotes the class of $L^{2}$-functions in $\Omega$ such that for all distribution derivatives up to $m$ belongs to $L^{2}(\Omega)$;

$H_{l o c}^{m}(\Omega)=\left\{f(x) \mid\right.$ for any compact set $\left.K \subset \Omega, f \in H^{m}(K)\right\}$;

$H_{0}^{1}(\Omega)$ denotes the completion of $C_{0}^{\infty}(\Omega)$ with respect to the norm $\left(\int_{\Omega}\left(|f|^{2}+|\operatorname{grad} f|^{2}\right) d x\right)^{1 / 2}$.

Next we shall state the conditions required in the theorems.

(A1) each $a_{i j}(x) \in C^{2}(\Omega)$ is a real-valued function;

(A2) $a_{i j}(x)=a_{j i}(x)$;

(A3) there exists a constant $G_{1} \geq 1$ such that for any $x \in \Omega$ and any $\xi \in \mathbb{C}^{n}$ we have

$$
C_{1}^{-1}|\xi|^{2} \leq\langle A(x) \xi, \bar{\xi}\rangle \leq C_{1}|\xi|^{2} ;
$$

(A4) $a_{i j}(x) \rightarrow \delta_{i j}$ as $|x| \rightarrow \infty$;

(A5) $\partial_{l} a_{i j}(x)=o\left(r^{-1}\right)$ as $|x| \rightarrow \infty$;

(A6) $\partial_{m} \partial_{l} a_{i j}(x)=o\left(r^{-1}\right)$ as $|x| \rightarrow \infty$.

(B1) each $b_{i}(x) \in C^{1}(\Omega)$ is a real-valued function;

(B2) $|B(x) A(x) x|=O(1)$ as $|x| \rightarrow \infty$.

(C1) $q_{1}(x)$ is a real-valued function;

(C2) for any $w \in H_{l o c}^{1}(\Omega)$ we have $q_{1}|w|^{2} \in L_{l o c}^{1}(\Omega)$;

(C3) for any $w \in H_{l o c}^{1}(\Omega)$ we have $\left|\operatorname{grad} q_{1}\right||w|^{2} \in L_{l o c}^{1}(\Omega)$;

(C4) there exist some real-valued function $\gamma(x)$ and some constant $0<r_{0}<2$ such that

$$
\begin{gathered}
\left|\gamma(x)-\gamma_{0}\right|+r^{-1 / 2}\left|\partial_{r} \gamma(x)\right|+\left|\operatorname{grad} \gamma(x)-\hat{x} \partial_{r} \gamma(x)\right|=o(1) \text { as } r \rightarrow \infty, \\
\quad \underset{r \rightarrow \infty}{\lim \sup _{r \rightarrow \infty}}\left\{r\left\langle\hat{x}, A(x) \operatorname{grad} q_{1}(x)\right\rangle+\gamma(x) q_{1}(x)\right\}<\infty .
\end{gathered}
$$

(D1) $q_{2}(x)$ is a complex-valued function;

(D2) $q_{2}(x)=O\left(r^{-1 / 2}\right)$ as $r \rightarrow \infty$.

(E) there exists some constant $R_{0}>0$ such that $\Omega \supset\left\{x|| x \mid>R_{0}\right\}$.

We shall consider

$$
\left(^{*}\right)\left\{\begin{array}{l}
-\langle D, A D u\rangle+\left(q_{1}+q_{2}\right) u=\lambda u \text { in } \Omega, \\
u \in H_{\text {loc }}^{2}(\Omega), \\
\operatorname{supp}[u] \text { is not a compact set in } \bar{\Omega} \text { (closure of } \Omega \text { ), }
\end{array}\right.
$$


where $\lambda$ is a real constant. Now we have

Theorem $\mathbb{1} .1$. Let $u$ satisfy $\left(^{*}\right)$, and let conditions $(A),(B),(C)$, (D), (E) hold. Then for any $\mu>0$ satisfying

$$
\begin{aligned}
(\#): \gamma_{0}\left(\mu^{2}+\lambda\right)>\lim \sup _{r \rightarrow \infty}\left\{r\left\langle\hat{x}, A(x) \operatorname{grad} q_{1}\right\rangle+\gamma(x) q_{1}(x)\right. \\
\left.+(4 \mu)^{-1} r\left|q_{2}(x)\right|^{2}+\left(2-\gamma_{0}\right)^{-1}|B(x) A(x) x|^{2}\right\},
\end{aligned}
$$

we have

$$
\lim _{R \rightarrow \infty} e^{2 \mu R} \int_{S(R)}\left[|\langle A D u, \hat{x}\rangle|^{2}+\left(1+\left(q_{1}\right)_{-}\right)|u|^{2}\right] d S=\infty
$$

Theorem 1.2. Besides the conditions assumed in Theorem 1.1, we assume that $q_{1}(x)$ satisfies the following:

$(F)$ for any $\varepsilon>0$ there exists some $C_{2}>0$ such that for any $w \in H_{0}^{1}(\Omega)$ we have

$$
\int_{\Omega}\left(q_{1}\right)-|w|^{2} d x \leq \varepsilon \int_{\Omega}|\operatorname{grad} w|^{2} d x+\left.C_{2} \int_{\Omega} i w\right|^{2} d x
$$

Then we have for $u$ and $\mu$ given in Theorem 1.1

and

$$
\lim _{R \rightarrow \infty} e^{2 \imath \ell R} \int_{B(R, R+1)}|u|^{2} d x=\infty
$$

$$
\int_{\Omega} e^{2 \mu r}|u|^{2} d x=\infty
$$

Remark 1. 1. Mochizuki [7] treated the case $\gamma(x)=\gamma_{0}\langle A \hat{x}, \hat{x}\rangle$.

Now we shall consider a more special case:

$$
(* *):\left\{\begin{array}{l}
-\langle D, D u\rangle+\left(q_{1}+q_{2}\right) u=\lambda u \text { in } \Omega \\
u \in H_{l o c}^{2}(\Omega), \\
\operatorname{supp}[u] \text { is not a compact set in } \bar{\Omega}
\end{array}\right.
$$

where $\lambda$ is a real constant. Here we can weaken (C3) as follows:

Theorem 1. 3. Let u satisfy (**). We assume conditions $(B),(C)$, (D), (E) with $a_{i j}(x)=\delta_{i j}$ except for (C3). Instead of (C3) we assume $(C 3)^{\prime}:$ for any $w \in H_{l o c}^{1}(\Omega)$ we have $\left(\partial_{r} q_{1}\right)|w|^{2} \in L_{l o c}^{1}(\Omega)$.

Then for any $\mu>0$ satisfying

$$
(\# \#): \gamma_{0}\left(\mu^{2}+\lambda\right)>\lim \sup _{r \rightarrow \infty}\left\{r \partial_{r} q_{1}(x)+\gamma(x) q_{1}(x)\right.
$$




$$
\left.+(4 \mu)^{-1} r\left|q_{2}(x)\right|^{2}+\left(2-\gamma_{0}\right)^{-1}|B(x) x|^{2}\right\}
$$

we have

$$
\lim _{R \rightarrow \infty} e^{2 \mu R} \int_{S(R)}\left[|\langle D u, \hat{x}\rangle|^{2}+\left(1+\left(q_{1}\right)_{-}\right)|u|^{2}\right] d S=\infty
$$

Theorem 1.4. Besides the conditions given in Theorem 1.3, we assume $(F)$, which is given in Theorem 1.2. Then for $u$ and $\mu$ given in Theorem 1.3 we have

$$
\lim _{R \rightarrow \infty} e^{2 \mu R} \int_{B(R, R+1)}|u|^{2} d x=\infty
$$

and

$$
\int_{\Omega} e^{2 \mu r}|u|^{2} d x=\infty
$$

As a special case of Theorem 1.4, we have

Theorem 1.5. We assume that $q_{1}(x)$ satisfies $(C 1),(C 2),(C 3)^{\prime},(F)$ and $\lim \sup \left(r \partial_{r} q_{1}+\gamma_{0} q_{1}\right) \leq 0$ for some constant $0<\gamma_{0}<2, q_{2}(x)$ is a complexvalued function satisfying $q_{2}(x)=o\left(r^{-1 / 2}\right)$ as $r \rightarrow \infty, b_{i}(x) \in C^{1}(\Omega)$ is a realvalued function $(1 \leq i \leq n)$ satisfying $\partial_{i} b_{j}(x)-\partial_{j} b_{i}(x)=o\left(r^{-1}\right)$ as $r \rightarrow \infty$ and (E) holds. Then for $u$ satisfying $\left(^{* *}\right)$ and for any $\varepsilon>0$ we have

$$
\begin{aligned}
& \lim _{R \rightarrow \infty} e^{2(1+\varepsilon) \sqrt{|\lambda|} R} \int_{B(R, R+1)}|u|^{2} d x=\infty, \text { if } \lambda<0, \\
& \lim _{R \rightarrow \infty} e^{\varepsilon R} \int_{B(R, R+1)}|u|^{2} d x=\infty, \quad \text { if } \lambda \geq 0 .
\end{aligned}
$$

Lastly we shall consider the most special case:

$$
(* * *):\left\{\begin{array}{l}
-\Delta u+\left(q_{1}+q_{2}\right) u=\lambda u \text { in } \Omega, \\
u \in H_{l o c}^{2}(\Omega), \\
\operatorname{supp}[u] \text { is not a compact set in } \bar{\Omega},
\end{array}\right.
$$

where $\lambda$ is a real constant and $\Delta$ is a Laplacian in $\boldsymbol{R}^{n}$. Now we can also weaken (C3) and (C4) as follows:

Theorem 1. 6. Let u satisfy (***). We assume conditions $(C),(D)$, $(E)$ except for (C3) and (C4). Instead of (C3) and (C4) we assume $(C 3)^{\prime}$ and 
$(C 4)^{\prime}$ : there exist some real-valued function $\gamma(x)$ and some constants $0<\gamma_{0} \leq 2,0<\alpha<2$ such that $\gamma(x)+\left|\operatorname{grad} \gamma(x)-\hat{x} \partial_{r} \gamma(x)\right|^{\alpha} \leq 2$ for $r>R_{0}$,

$\left|\gamma(x)-\gamma_{0}\right|+r^{-1 / 2}\left|\partial_{r} \gamma(x)\right|+\left|\operatorname{grad} \gamma(x)-\hat{x} \partial_{r} \gamma(x)\right|=o(1)$ as $r \rightarrow \infty$,

$$
\lim _{r \rightarrow \infty} \sup \left\{r \partial_{r} q_{1}(x)+r(x) q_{1}(x)\right\}<\infty .
$$

Then for any $\mu>0$ satisfying

$$
(\# \#): \gamma_{0}\left(\mu^{2}+\lambda\right)>\lim \sup _{r \rightarrow \infty}\left\{r \partial_{r} q_{1}(x)+r(x) q_{1}(x)+(4 \mu)^{-1} r\left|q_{2}(x)\right|^{2}\right\},
$$

we have

$$
\lim _{R \rightarrow \infty} e^{2 \mu R} \int_{S(R)}\left[\left|\partial_{r} u\right|^{2}+\left(1+\left(q_{1}\right)_{-}\right)|u|^{2}\right] d S=\infty \text { 。 }
$$

Theorem 1.7. Besides the conditions given in Theorem 1.6, we assume $(F)$. Then for $u$ and $\mu$ given in Theorem 1.6 we have

$$
\lim _{R \rightarrow \infty} e^{2 \mu R} \int_{B(R, R+1)}|u|^{2} d x=\infty
$$

and

$$
\int_{\Omega} e^{2 \mu r}|u|^{2} d x=\infty
$$

Remark 1.2. If $0<\gamma_{0}<2$ and $a_{i j}(x)=\delta_{i j}$, (C4)' is the same condition as (C4).

\section{§2. Proof of Theorem 1.1}

We shall begin to give the following definition of which meaning is shown in Lemma 2.1. In $\S 2$ we assume (A), (B), (C), (D) and (E).

Definition 2. 1. Let $u(x)$ satisfy $\left(^{*}\right)$. For smooth real-valued functions $\rho=\rho(r), f=f(r)$ and $g=g(x)$, let

$$
\begin{aligned}
& v(x)=e^{\rho(r)} u(x), \\
& k_{1}(x)=-\left\{\rho^{\prime}(r)\right\}^{2}\langle A(x) \hat{x}, \hat{x}\rangle, \\
& k_{2}(x)=\rho^{\prime \prime}(r)\langle A(x) \hat{x}, \hat{x}\rangle+\rho^{\prime}(r) \operatorname{div}\{A(x) \hat{x}\}
\end{aligned}
$$

and 


$$
\begin{aligned}
F(t ; \rho, f, g) & =\int_{S(t)}\left[f \left\{2|\langle\hat{x}, A D v\rangle|^{2}-\langle\hat{x}, A \hat{x}\rangle\langle\langle D v, A \overline{D v}\rangle\right.\right. \\
& \left.\left.\left.+\left(q_{1}-\lambda+k_{1}\right)|v|^{2}\right)\right\}+g \operatorname{Re}[\langle\hat{x}, A \overline{D v}\rangle v]\right] d S,
\end{aligned}
$$

where $\operatorname{Re}[w]$ means the real part of $w$.

Lemma 2.1. For $t>s>R_{0}$ we have

$$
\begin{aligned}
F( & (; \rho, f, g)-F(s ; \rho, f, g) \\
= & \int_{B(s, t)}\left[2\left(2 \rho^{\prime} f+f^{\prime}-r^{-1} f\right)|\langle A D v, \hat{x}\rangle|^{2}+\left(2 r^{-1} f+g\right.\right. \\
- & \left.f \operatorname{div}(A \hat{x})-\langle\hat{x}, A \hat{x}\rangle f^{\prime}\right)\langle A D v, \overline{D v}\rangle+2 r^{-1} f\left(|A D v|^{2}\right. \\
& -\langle A D v, \overline{D v}\rangle)+2 f \operatorname{Re}[\langle\hat{x},(\langle A D v, \operatorname{grad}\rangle A) \overline{D v}\rangle] \\
& -f\langle(\langle\hat{x}, A \operatorname{grad}\rangle A) D v, \overline{D v}\rangle+2 \operatorname{Re}\left[\left(f\left(q_{2}+k_{2}\right)+g \rho^{\prime}\right.\right. \\
& \left.\left.+2^{-1} \partial_{r} g\right)\langle\hat{x}, \overline{D v}\rangle v\right] \\
- & \left.2 f \operatorname{Im}[\langle B A \hat{x}, A \overline{D v}\rangle v]+\operatorname{Re}\left[\left\langle\left(\operatorname{grad} g-\hat{x} \partial_{r} g\right), A \overline{D v}\right\rangle\right]\right] \\
& +\left\{\left(q_{1}-\lambda+k_{1}\right)\left(g-f \operatorname{div}(A \hat{x})-f^{\prime}\langle\hat{x}, A \hat{x}\rangle\right)\right. \\
& \left.\left.-f\left\langle\hat{x}, A \operatorname{grad}\left(q_{1}+k_{1}\right)\right\rangle+g\left(\operatorname{Re}\left[q_{2}\right]+k_{2}\right)\right\}|v|^{2}\right] d x,
\end{aligned}
$$

where $\operatorname{Im}[w]$ means the imaginary part of $w$.

Proof. By Definition 2.1 $v(x) \in H_{l o c}^{2}(\Omega)$ and $v(x)$ satisfies for $r>R_{0}$

$$
-\langle D, A D v\rangle+2 \rho^{\prime}\langle\hat{x}, A D v\rangle+\left\{\left(q_{1}-\lambda+k_{1}\right)+\left(q_{2}+k_{2}\right)\right\} v=0 .
$$

Then we have

$$
\begin{aligned}
& \operatorname{Re} \int_{B(s, t)}\left[-\langle D, A D v\rangle+2 \rho^{\prime}\langle\hat{x}, A D v\rangle+\left\{\left(q_{1}-\lambda+k_{1}\right)+\left(q_{2}+k_{2}\right)\right\} v\right] \\
& \quad \times[2 f(r)\langle\hat{x}, A \overline{D v}\rangle+g(x) \bar{v}] d x=0 .
\end{aligned}
$$

In the left side of the above equation we use the integration by parts such as

$$
\int_{B(s, t)} \partial_{i} w d x=\int_{S(t)} \hat{x}_{i} w d S-\int_{S(s)} \hat{x}_{i} w d S \quad \text { for } w, \partial_{i} w \in L_{l o c}^{1}(\Omega)
$$

(Cf. Lemma 4.1). Noting

$$
\begin{aligned}
& D_{i} D_{j}-D_{j} D_{i}=\sqrt{-1}\left(\partial_{i} b_{j}(x)-\partial_{j} b_{i}(x)\right), \\
& 2 \operatorname{Re}\left[\left\langle D_{i} D v, A \overline{D v}\right\rangle\right]=\partial_{i}(\langle A D v, \overline{D v}\rangle)-\left\langle\left(\partial_{i} A\right) D v, \overline{D v}\right\rangle,
\end{aligned}
$$

we have the assertion.

Lemma 2. 2. Let $1-2^{-1} \gamma_{0}<l<1$. Then for any $\mu>0$ satisfying (\#) 
there exists some $R_{1} \geq R_{0}$ such that for any $t>s>R_{1}$ and any $m \geq 1$, we have

$$
\begin{aligned}
& F\left(t ; \mu r+m r^{l}, 1, \operatorname{div}(A \hat{x})-r(x) r^{-1}\right) \\
& \geq F\left(s ; \mu r+m r^{l}, 1, \operatorname{div}(A \hat{x})-r(x) r^{-1}\right) .
\end{aligned}
$$

Proof. Let $\mu>0$ satisfy (\#). By (B2) we can find some $0<\delta<2-\gamma_{0}$ such that

$$
\begin{aligned}
& \gamma_{0}\left(\mu^{2}+\lambda\right)>\lim \sup _{r \rightarrow \infty}\left\{r\left\langle\hat{x}, A \operatorname{grad} q_{1}\right\rangle+\gamma q_{1}+(4 \mu)^{-1} r \mid q_{2} !^{2}\right. \\
& \left.\quad+\delta^{-1}|B A x|^{2}\right\} .
\end{aligned}
$$

Let

$$
\rho(r)=\mu r+m r^{l}, f(r)=1 \text { and } g(x)=\operatorname{div}(A(x) \hat{x})-r(x) r^{-1},
$$

where $m \geq 1$ and $1-2^{-1} \gamma_{0}<l<1$ are constants. Let each $\varepsilon_{i}(r) \quad(i=1,2$, ...) be a positive function for $r>0$ which tends to 0 as $r \rightarrow \infty$ 。 Noting

$$
\begin{gathered}
|\langle A \hat{x}, \hat{x}\rangle-1|+r|\langle\hat{x}, A \operatorname{grad}(\langle A \hat{x}, \hat{x}\rangle)\rangle|+r^{1 / 2}\left|\partial_{r} g\right| \\
+r\left|\operatorname{grad} g-\hat{x} \partial_{r} g\right| \leq \varepsilon_{1}(r), \\
\operatorname{div}(A \hat{x})=O\left(r^{-1}\right) \quad \text { as } r \rightarrow \infty,
\end{gathered}
$$

we have the following by direct calculation.

$$
\begin{aligned}
& 2\left(2 \rho^{\prime} f+f^{\prime}-r^{-1} f\right)|\langle A D v, \hat{x}\rangle|^{2}=r^{-1}\left(4 \mu r+4 m l r^{l}-2\right)|\langle A D v, \hat{x}\rangle|^{2}, \\
& \left(2 r^{-1} f+g-f \operatorname{div}(A \hat{x})-\langle\hat{x}, A \hat{x}\rangle f^{\prime}\right)\langle A D v, \overline{D v}\rangle \\
& \quad \geq r^{-1}\left(2-\gamma_{0}-\varepsilon_{2}(r)\right)\langle A D v, \overline{D v}\rangle, \\
& 2 r^{-1} f\left(|A D v|^{2}-\langle A D v, \overline{D v}\rangle\right) \geq-\varepsilon_{3}(r) r^{-1}\langle A D v, \overline{D v}\rangle, \\
& 2 f \operatorname{Re}[\langle\hat{x},(\langle A D v, \operatorname{grad}\rangle A) \overline{D v}\rangle] \geq-\varepsilon_{4}(r) r^{-1}\langle A D v, \overline{D v}\rangle, \\
& -f\langle(\langle\hat{x}, A \operatorname{grad}\rangle A) D v, \overline{D v}\rangle \geq-\varepsilon_{5}(r) r^{-1}\langle A D v, \overline{D v}\rangle, \\
& \left.2 \operatorname{Re}\left[\left\{f\left(q_{2}+k_{2}\right)+g \rho^{\prime}+2^{-1} \partial_{r} g\right\}\langle\hat{x}, A \overline{D v}\rangle\right]\right] \\
& \geq-r^{-1}\left(4 \mu r+m l r^{l}\right)|\langle\hat{x}, A D v\rangle|^{2}-r^{-1}\left\{(4 \mu)^{-1} r\left|q_{2}\right|^{2}\right. \\
& \left.\quad+m l r^{l-1} \varepsilon_{6}(r)+\varepsilon_{7}(r)\right\}|v|^{2}, \\
& -2 f \operatorname{Im}[\langle B A \hat{x}, A \overline{D v}\rangle v] \geq-\delta r^{-1}\langle A D v, \overline{D v}\rangle-r^{-1}\left(\delta^{-1}|B A x|^{2}\right. \\
& \left.\quad+\varepsilon_{8}(r)\right)|v|^{2}, \\
& \operatorname{Re}\left[\left\langle\left(\operatorname{grad} g-\hat{x} \partial_{r} g\right), A \overline{D v}\right\rangle v\right] \geq-\varepsilon_{9}(r) r^{-1}\left(\langle A D v, \overline{D v}\rangle+|v|^{2}\right), \\
& \left(q_{1}-\lambda+k_{1}\right)\left(g-f \operatorname{div}(A \hat{x})-f^{\prime}\langle\hat{x}, A \hat{x}\rangle\right)-f\left\langle\hat{x}, A \operatorname{grad}\left(q_{1}+k_{1}\right)\right\rangle \\
& \quad=r^{-1}\left(\gamma \rho^{\prime 2}\langle A \hat{x}, \hat{x}\rangle+2 r \rho^{\prime} \rho^{\prime \prime}\langle A \hat{x}, \hat{x}\rangle^{2}+r \rho^{\prime 2}\langle\hat{x}, A \operatorname{grad}(\langle A \hat{x}, \hat{x}\rangle)\rangle\right. \\
& \left.\quad+\gamma \lambda-r\left\langle\hat{x}, A \operatorname{grad} q_{1}\right\rangle-\gamma q_{1}\right) \\
& \geq r^{-1}\left[\left\{\gamma_{0}\left(\mu^{2}+\lambda\right)-\left(r\left\langle\hat{x}, A \operatorname{grad} q_{1}\right\rangle+\gamma q_{1}\right)-\varepsilon_{10}(r)\right\}\right.
\end{aligned}
$$




$$
\begin{aligned}
+m^{2} l^{2}\left(2 l+\gamma_{0}-2-\varepsilon_{11}(r)\right) r^{2 l-2} \\
\left.+2 \mu m l\left(l+\gamma_{0}-1-\varepsilon_{12}(r)\right) r^{l-1}\right], \\
g\left(\operatorname{Re}\left[q_{2}\right]+k_{2}\right) \geq-\varepsilon_{13}(r) r^{-1}\left(1+2 \mu m l r^{l-1}\right) .
\end{aligned}
$$

Then by Lemma 2. 1, we have

$$
\begin{aligned}
F(t ; \mu r & \left.+m r^{l}, 1, \operatorname{div}(A \hat{x})-\gamma(x) r^{-1}\right) \\
& -F\left(s ; \mu r+m r^{l}, 1, \operatorname{div}(A \hat{x})-\gamma(x) r^{-1}\right) \\
\geq & \int_{B(s, t)} r^{-1}\left[\left(3 m l r^{l}-2\right)|\langle A D v, \hat{x}\rangle|^{2}\right. \\
& +\left(2-\gamma_{0}-\delta-\varepsilon_{14}(r)\right)\langle A D v, \overline{D v}\rangle \\
& +\left\{\gamma_{0}\left(\mu^{2}+\lambda\right)-\left(r\left\langle\hat{x}, A \operatorname{grad} q_{1}\right\rangle\right.\right. \\
& \left.\left.+\gamma(x) q_{1}+(4 \mu)^{-1} r\left|q_{2}\right|^{2}+\delta^{-1}|B A x|^{2}\right)-\varepsilon_{14}(r)\right\}|v|^{2} \\
& +m^{2} l^{2}\left(2 l+\gamma_{0}-2-\varepsilon_{14}(r)\right) r^{2 l-2}|v|^{2} \\
& \left.+2 \mu m l\left(l+\gamma_{0}-1-\varepsilon_{14}(r)\right) r^{l-1}|v|^{2}\right] d x .
\end{aligned}
$$

Noting

$$
l+\gamma_{0}-1=2^{-1}\left(2 l+\gamma_{0}-2\right)+2^{-1} \gamma_{0}>0,
$$

there exists some $R_{1} \geq R_{0}$ such that for any $r \geq R_{1}$ and $m \geq 1$, we have

$$
\begin{aligned}
& 3 m l R_{1}^{l}-2 \geq 0, \\
& 2-\gamma_{0}-\delta-\varepsilon_{14}(r) \geq 0, \\
& \gamma_{0}\left(\mu^{2}+\lambda\right)-\left(r\left\langle\hat{x}, A \text { grad } q_{1}\right\rangle+\gamma(x) q_{1}+(4 \mu)^{-1} r\left|q_{2}\right|^{2}\right. \\
& \left.\quad \quad+\delta^{-1}|B A x|^{2}\right)-\varepsilon_{14}(r) \geq 0, \\
& 2 l+\gamma_{0}-2-\varepsilon_{14}(r) \geq 0, \\
& l+\gamma_{0}-1-\varepsilon_{14}(r) \geq 0 .
\end{aligned}
$$

Therefore we have the assertion.

Proof of Theorem 1. 1. If $\lim \sup _{r \rightarrow \infty}\left\{r\left\langle\hat{x}, A\right.\right.$ grad $\left.\left.q_{1}\right\rangle+r q_{1}\right\}>-\infty$, then by (B2) and (D2)

$$
\begin{aligned}
\gamma_{0}\left(\mu^{2}+\lambda\right) & -\lim _{r \rightarrow \infty} \sup \left\{r \hat{x}, A \operatorname{grad} q_{1}\right\rangle+\gamma q_{1}+(4 \mu)^{-1} r\left|q_{2}\right|^{2} \\
& \left.+\left(2-\gamma_{0}\right)^{-1}|B A x|^{2}\right\}
\end{aligned}
$$

is a continuous function of $\mu>0$. Therefore for any $\mu>0$ satisfying (\#), we can find $\mu^{\prime}$ such that $0<\mu^{\prime}<\mu$ and $\mu^{\prime}$ satisfies (\#) also. This conclusion also holds in case $\lim _{r \rightarrow \infty} \sup \left\{r\left\langle\hat{x}, A \operatorname{grad} q_{1}\right\rangle+\gamma q_{1}\right\}=-\infty$. Noting the fact mentioned above, we have only to show that for any $\mu^{\prime}>0$ satisfying (\#) we have

$$
\lim _{R \rightarrow \infty} \inf ^{2 \mu^{\prime} R} \int_{S(R)}\left[|\langle A D u, \hat{x}\rangle|^{2}+\left(1+\left(q_{1}\right)_{-}\right)|u|^{2}\right] d S>0 .
$$


We shall prove above by contradiction. So we assume that this is not true. Then there exists some $\mu_{0}>0$ satisfying (\#) such that

$$
\liminf _{R \rightarrow \infty} e^{2 \mu_{0} R} \int_{S(R)}\left[|\langle A D u, \hat{x}\rangle|^{2}+\left(1+\left(q_{1}\right)-|u|^{2}\right] d S=0 .\right.
$$

By the remarks given at the beginning of this proof, we can find $0<\mu<\mu_{0}$ satisfying (\#). By Definition 2.1, for given $m \geq 1$ and $1-2^{-1} \gamma_{0}<l<1$ there exists some constant $C_{3}>0$ such that for any $t>R_{0}$ we have

$$
\begin{aligned}
F(t ; \mu r & \left.+m r^{l}, 1, \operatorname{div}(A \hat{x})-\gamma r^{-1}\right) \\
& =e^{2 \rho(t)} \int_{S(t)}\left[2|\langle\hat{x}, A D u\rangle|^{2}+\langle\hat{x}, A \hat{x}\rangle\left\{-\langle D u, A \overline{D u}\rangle+\left(\hat{\lambda}-q_{1}\right)|u|^{2}\right.\right. \\
& \left.+\left(\operatorname{div}(A \hat{x})-\gamma r^{-1}\right) \rho^{\prime}|u|^{2}\right\}+2 \rho^{2}\langle\hat{x}, A \hat{x}\rangle^{2}|u|^{2} \\
& +\left(2 \rho^{\prime}\langle\hat{x}, A \hat{x}\rangle+\operatorname{div}(A \hat{x})-\gamma r^{-1}\right) \operatorname{Re}[\langle\hat{x}, A \overline{D u}\rangle u] d S \\
& \leq C_{3} e^{2 \mu t+2 m t^{t}} \int_{S(t)}\left[|\langle\hat{x}, A D u\rangle|^{2}+\left(1+\left(q_{1}\right)_{-}\right)|u|^{2}\right] d S,
\end{aligned}
$$

where $\rho=\mu r+m r^{l}$. Then noting $l<1$ and $\mu<\mu_{0}$ we have

$$
\lim _{t \rightarrow \infty} \inf F\left(t ; \mu r+m r^{l}, 1, \operatorname{div}(A \hat{x})-\gamma r^{-1}\right) \leq 0 .
$$

So letting $t \rightarrow \infty$ along suitable sequence in Lemma 2.2, we have for any $s \geq R_{1}$ and any $m \geq 1$

$$
F\left(s ; \mu r+m r^{l}, 1, \operatorname{div}(A \hat{x})-\gamma r^{-1}\right) \leq 0 .
$$

On the other hand, since $\operatorname{supp}[u]$ is not a compact set in $\bar{\Omega}$, there exists some $R_{2} \geq R_{1}$ such that

$$
\int_{S\left(R_{2}\right)}|\langle\hat{x}, A \hat{x}\rangle|^{2}|u|^{2} d S>0 .
$$

Since $e^{-2 \mu R_{2}-2 m R_{2}^{l}} F\left(R_{2} ; \mu r+m r^{l}, 1, \operatorname{div}(A \hat{x})-\gamma r^{-1}\right)$ is a quadratic in $m$ of which coefficient of $m^{2}$ is $2 l^{2} R_{2}^{2(l-1)} \int_{S\left(R_{L}\right)}|\langle\hat{x}, A \hat{x}\rangle|^{2}|u|^{2} d S>0$, then there exists some $m_{0} \geq 1$ such that

$$
F\left(R_{2} ; \mu r+m_{0} r^{l}, 1, \operatorname{div}(A \hat{x})-\gamma r^{-1}\right)>0,
$$

which leads to the contradiction.

\section{§3. Proof of Theorem 1.2}

Lemma 3.1. Let $\Omega \supset\left\{x|| x \mid>R_{0}\right\}$ be a domain in $\mathbb{R}^{n}$, and each $b_{i}(x)$ $(i=1, \ldots, n)$ be real-valued function on $\Omega$. Let $V(x)$ satisfy the following: 
for any $\varepsilon>0$ there exists some $G_{4}>0$ such that for any $w \in H_{0}^{1}(\Omega)$

$$
\int_{\Omega}|V(x)||w(x)|^{2} d x \leq \varepsilon \int_{\Omega}|\operatorname{grad} w|^{2} d x+C_{4} \int_{\Omega}|w(x)|^{2} d x .
$$

Let $\zeta(t)$ be a smooth function on $(-\infty, \infty)$ satisfying $0 \leq \zeta(t) \leq 1$ for $-\infty<t<\infty, \zeta(t)=1$ for $1 / 3 \leq t \leq 2 / 3$ and $\operatorname{supp}[\zeta] \subset(0,1)$. And let $\zeta_{R}(t)=\zeta(t-R)$. Then for any $\varepsilon>0$ there exists some constant $G_{5}>0$ such that for any $w \in H_{l o c}^{1}(\Omega)$ and any $R>R_{0}$, we have

$$
\int_{\Omega} \zeta_{R}^{2}(|x|)|V(x)||w(x)|^{2} d x \leq \varepsilon \int_{\Omega} \zeta_{R}^{2}|D w|^{2} d x+C_{5} \int_{B(R, R+1)}|w|^{2} d x .
$$

Proof. Let $w^{(\eta)}(x)=\left\{|w(x)|^{2}+\eta^{2}\right\}^{1 / 2}$ for $\eta>0$ and $w \in H_{l o c}^{1}(\Omega)$. Then $w^{(\eta)} \in H_{l o c}^{1}(\Omega)$ and $\left|\operatorname{grad} w^{(\eta)}\right| \leq|D w|$, because of $w^{(\eta)} \operatorname{grad} w^{(\eta)}=\operatorname{Re}$ $[\bar{w} \operatorname{grad} w]=\operatorname{Re}[\bar{w} D w]$ and $|w| \leq\left|w^{(\eta)}\right|$. Then by $\zeta_{R} w^{(\eta)} \in H_{0}^{1}(\Omega)$ for $R>R_{0}$, we have

$$
\begin{aligned}
& \int_{\Omega} \zeta_{R}^{2}|v|\left|w^{(\eta)}\right|^{2} d x \leq \varepsilon \int_{\Omega}\left|\operatorname{grad}\left(\zeta_{R} w^{(\eta)}\right)\right|^{2} d x+C_{4} \int_{\Omega}\left|\zeta_{R} w^{(\eta)}\right|^{2} d x \\
& \quad \leq 2 \varepsilon \int_{\Omega} \zeta_{R}^{2}|D w|^{2} d x+\left(C_{4}+2 \varepsilon \max _{0 \leq t \leq 1}\left(\zeta^{\prime}(t)\right)^{2}\right) \int_{B(R, R+1)}\left|w^{(\eta)}\right|^{2} d x .
\end{aligned}
$$

Letting $\eta \rightarrow 0$, we have the assertion. (This proof is the same one given by Eastham-Kalf [3, p. 249].)

Lemma 3. 2. Let $\Omega \supset\left\{x|| x \mid>R_{0}\right\}$ be a domain in $\mathbb{R}^{n}$, and let $\zeta_{R}(t)$ be the same as given in Lemma 3.1. Let $A(x)=\left(a_{i j}(x)\right), b_{i}(x)(i=1$, $\ldots, n)$ and $V(x)$ satisfy the following:

(1): there exists some constant $C_{6} \geq 1$ such that for any $x \in \Omega$ and any $\xi \in \boldsymbol{C}^{n}$

$$
C_{6}^{-1}|\xi|^{2} \leq\langle A(x) \xi, \bar{\xi}\rangle \leq C_{6}|\xi|^{2},
$$

(2): $\operatorname{div}(A \hat{x})$ is bounded on $\Omega$,

(3): each $b_{i}(x) \in C^{1}(\Omega)$ is a real-valued function,

(4): $V(x)$ is a complex-valued function on $\Omega$,

(5): for any $\varepsilon>0$ there exists some $C_{7}>0$ such that for any $w \in H_{0}^{1}(\Omega)$

$$
\int_{\Omega}(\operatorname{Re} V)-(x)|w(x)|^{2} d x \leq \varepsilon \int_{\Omega}|\operatorname{grad} w|^{2} d x+C_{7} \int_{\Omega}|w|^{2} d x .
$$

Let $u \in H_{\text {loc }}^{2}(\Omega)$ satisfy

$$
-\langle D, A D u\rangle+V u=0 \text { in } \Omega \text { 。 }
$$

Then there exists some constant $C_{8}>0$ such that for any $R>R_{0}$ we have 


$$
\int_{\Omega} \zeta_{R}^{2}\left\{|D u|^{2}+(\operatorname{Re} V)-|u|^{2}\right\} d x \leq C_{8} \int_{B(R, R+1)}|u|^{2} d x
$$

Proof. By Lemma 3. 1, for any $\varepsilon>0$ there exists some $C_{5}>0$ such that

$$
\int_{\Omega} \zeta_{R}^{2}(\operatorname{Re} V)-|u|^{2} d x \leq \varepsilon \int_{\Omega} \zeta_{R}^{2}|D u|^{2} d x+C_{5} \int_{B(R, R+1)}|u|^{2} d x .
$$

So we have only to show that there exists some constant $C_{9}>0$ such that for any $R>R_{0}$

$$
\int_{\Omega} \zeta_{R}^{2}|D u|^{2} d x \leq C_{9} \int_{B(R, R+1)}|u|^{2} d x
$$

By integration by parts we have

$$
\begin{aligned}
0 & =\operatorname{Re} \int_{\Omega} \zeta_{R}^{2}[-\langle D, A D u\rangle+V u] \bar{u} d x \\
& =\int_{\Omega}\left[\zeta_{R}^{2}\left\{\langle A D u, \overline{D u}\rangle+(\operatorname{Re} V)|u|^{2}\right\}-\left\{\zeta_{R} \zeta_{R}^{\prime} \operatorname{div}(A \hat{x})\right.\right. \\
& \left.\left.+\left(\zeta_{R} \zeta_{R}^{\prime}\right)^{\prime}\langle\hat{x}, A \hat{x}\rangle\right\}|u|^{2}\right] d x \\
& \geq \int_{\Omega} \zeta_{R}^{2}\left[C_{6}^{-1}|D u|^{2}-(\operatorname{Re} V)-|u|^{2}\right] d x-C_{10} \int_{B(R, R+1)}|u|^{2} d x \\
& \geq\left(C_{6}^{-1}-\varepsilon\right) \int_{\Omega} \zeta_{R}^{2}|D u|^{2} d x-\left(C_{5}+C_{10}\right) \int_{B(R, R+1)}|u|^{2} d x
\end{aligned}
$$

where we also use Lemma 3.1. So if we choose $\varepsilon$ to satisfy $0<\varepsilon<C_{6}^{-1}$, we have the assertion.

Now we prove Theorem 1.2.

Proof of Theorem 1.2. By Theorem 1.1, for any $\mu>0$ satisfying (\#) we have

$$
\lim _{R \rightarrow \infty} e^{2 \mu R} \int_{S(R)}\left[|\langle A D u, \hat{x}\rangle|^{2}+\left(1+\left(q_{1}\right)_{-}\right)|u|^{2}\right] d S=\infty .
$$

Fix $\mu>0$ satisfying (\#). Then for any $M>0$ there exists $R_{3}>R_{0}$ such that for any $t>R_{3}$

$$
\int_{S(t)}\left[|\langle A D u, \hat{x}\rangle|^{2}+\left(1+\left(q_{1}\right)_{-}\right)|u|^{2}\right] d S \geq M e^{-2 \mu t} .
$$

So we have for $R>R_{3}$

$$
M \int_{0}^{1} \zeta^{2}(t) d t \circ e^{-2 \mu(R+1)} \leq M \int_{R}^{R+1} \zeta_{R}^{2}(t) e^{-2 \mu t} d t
$$




$$
\begin{aligned}
& \leq \int_{B(R, R+1)} \zeta_{R}^{2}\left[|\langle A D u, \hat{x}\rangle|^{2}+\left(1+\left(q_{1}\right)_{-}\right)|u|^{2}\right] d x \\
& \leq C_{11}\left(\int_{\Omega} \zeta_{R}^{2}\left\{|D u|^{2}+\left(q_{1}\right)_{-}|u|^{2}\right\} d x+\int_{B(R, R+1)}|u|^{2} d x\right) \\
& \leq C_{12} \int_{B(R, R+1)}|u|^{2} d x,
\end{aligned}
$$

where we use Lemma 3.2 with $V(x)=q_{1}+q_{2}-\lambda$, which satisfies the condition given in Lemma 3.2. This means the former half of the statement of Theorem 1.2 holds. The latter half is easily obtained from

$$
\int_{\Omega} e^{2 \mu r}|u|^{2} d x \geq e^{2 \mu R} \int_{B(R, R+1)}|u|^{2} d x \text { for } R>R_{0} .
$$

\section{§4. Proofs of Theorems $1.3 \sim 1.7$}

If $a_{i j}(x)=\delta_{i j}$ in $\Omega$, we can weaken (C3) as (C3)'. (The author [10] and Mochizuki [7] neglected this condition, but Kalf [6] pointed out this in application of the Gauss's theorem (integration by parts).) We prepare the following:

Lemma 4. 1. Let $0<a<b$. We assume $w \in L^{1}(B(a, b))$ and $\partial_{r} w \in$ $L^{1}(B(a, b))$. Then for any $a<t<b, \int_{S(t)} w d S$ exists (by choosing suitable representative for w, if necessary) and for any $a<s<t<b$

$$
\int_{B(s, t)} \partial_{r} w d x=\int_{S(t)} w d S-\int_{S(s)} w d S-\int_{B(s, t)}(n-1) r^{-1} w d x .
$$

Proof. Let $j(\sigma) \in C_{0}^{\infty}(-\infty, \infty)$ satisfy the following: $j(\sigma)=0$ for $|\sigma| \geq 1, j(\sigma) \geq 0$ for $\sigma \in(-\infty, \infty)$ and $\int_{-\infty}^{\infty} j(\sigma) d \sigma=1$. Take $a^{\prime}, b^{\prime}$ arbitrarily to satisfy $a<a^{\prime}<b^{\prime}<b$, and let

$$
\begin{array}{ll}
h(r)=\int_{S(r)} w(x) d S & \text { for } a<r<b, \\
h_{\varepsilon}(\rho)=\varepsilon^{-1} \int_{B(a, b)} j\left(\varepsilon^{-1}(\rho-|x|)\right) w(x) d x & \text { for } a^{\prime}<\rho<b^{\prime},
\end{array}
$$

where $0<\varepsilon<\min \left\{a^{\prime}-a, b-b^{\prime}\right\}$. Since $h(r) \in L^{1}(a, b)$, for any $\eta>0$ there exists some $0<\delta<\min \left\{a^{\prime}-a, b-b^{\prime}\right\}$ such that for any $\xi$ satisfying $|\xi|<\delta$ we have 


$$
\int_{a^{\prime}}^{b^{\prime}}|h(r)-h(r+\xi)| d r<\eta
$$

We have for $a^{\prime}<\rho<b^{\prime}$

$$
h_{\varepsilon}(\rho)=\int_{\rho-\varepsilon}^{\rho+\varepsilon} \varepsilon^{-1} j\left(\varepsilon^{-1}(\rho-r)\right) h(r) d r=\int_{-1}^{1} j(\sigma) h(\rho-\varepsilon \sigma) d \sigma .
$$

Then for any $\eta>0$ there exists some $0<\delta<\min \left\{a^{\prime}-a, b-b^{\prime}\right\}$ such that for any $0<\varepsilon<\delta$

$$
\begin{aligned}
\int_{a^{\prime}}^{b^{\prime}}\left|h_{\varepsilon}(\rho)-h(\rho)\right| d \rho & \leq \int_{a^{\prime}}^{b^{\prime}} d \rho \int_{-1}^{1} j(\sigma)|h(\rho-\varepsilon \sigma)-h(\rho)| d \sigma \\
& =\int_{-1}^{1} j(\sigma) d \sigma \int_{a^{\prime}}^{b^{\prime}}|h(\rho-\varepsilon \sigma)-h(\rho)| d \rho<\eta .
\end{aligned}
$$

Next we have

$$
\begin{aligned}
\frac{d}{d \rho} h_{\varepsilon}(\rho) & =\varepsilon^{-1} \int_{B(a, b)} \partial_{\rho}\left\{j\left(\varepsilon^{-1}(\rho-|x|)\right)\right\} w(x) d x \\
& =-\varepsilon^{-1} \int_{B(a, b)} \partial_{r}\left\{j\left(\varepsilon^{-1}(\rho-r)\right)\right\} w(x) d x \\
& =\varepsilon^{-1} \int_{B(a, b)} j\left(\varepsilon^{-1}(\rho-r)\right)\left(\partial_{r} w+(n-1) r^{-1} w\right) d x,
\end{aligned}
$$

where we use the definition of distribution derivative $\partial_{r} w$. So by $\partial_{r} w+(n-1) r^{-1} w \in L^{1}(B(a, b))$ we have similarly

$$
\int_{a^{\prime}}^{b^{\prime}}\left|\frac{d}{d \rho} h_{\varepsilon}(\rho)-\int_{S(\rho)}\left(\partial_{r} w+(\mathrm{n}-1) r^{-1} w\right) d S\right| d \rho \rightarrow 0 \text { as } \varepsilon \rightarrow 0 .
$$

By $h_{\varepsilon}(\rho) \in C^{1}\left[a^{\prime}, b^{\prime}\right]$, for any $b^{\prime \prime}$ satisfying $a^{\prime}<b^{\prime \prime}<b^{\prime}$ there exists some $C_{13}>0$ such that for any $\rho$ satisfying $a^{\prime}<\rho<b^{\prime \prime}$ we have

$$
\begin{gathered}
\left|h_{\varepsilon}(\rho)-h_{\varepsilon^{\prime}}(\rho)\right|=\left(b^{\prime}-\rho\right)^{-1} \mid \int_{\rho}^{b^{\prime}} \frac{d}{d \sigma}\left\{\left(b^{\prime}-\sigma\right)\left(h_{\varepsilon}(\sigma)-h_{\varepsilon^{\prime}}(\sigma)\right\} d \sigma \mid\right. \\
\leq C_{13} \int_{a^{\prime}}^{b^{\prime}}\left\{\left|\frac{d}{d \sigma} h_{\varepsilon}(\sigma)-\frac{d}{d \sigma} h_{\varepsilon^{\prime}}(\sigma)\right|+\left|h_{\varepsilon}(\sigma)-h_{\varepsilon^{\prime}}(\sigma)\right|\right\} d \sigma \rightarrow 0
\end{gathered}
$$

as $\varepsilon, \varepsilon^{\prime} \rightarrow 0$. Because $b^{\prime \prime}$ is arbitrary and $\int_{a^{\prime}}^{b^{\prime}}\left|h_{\varepsilon}(\rho)-h(\rho)\right| d \rho \rightarrow 0$ as $\varepsilon \rightarrow 0$, we have $\lim _{\varepsilon \rightarrow 0} h_{\varepsilon}(\rho)=h(\rho)$ for any $\rho \in\left(a^{\prime}, b^{\prime}\right)$, where suitable representative of $w$ is chosen, if necessary. Since for any $s, t$ satisfying $a^{\prime}<s<t<b^{\prime}$

$$
\int_{s}^{t} \frac{d}{d \rho} h_{\varepsilon}(\rho) d \rho=h_{\varepsilon}(t)-h_{\varepsilon}(s),
$$

we have the assertion for $a^{\prime}<s<t<b^{\prime}$ by letting $\varepsilon \rightarrow 0$. Because $a^{\prime}$ 
and $b^{\prime}$ are arbitrary, we have the assertion.

Now we can show the proofs of Theorem 1.3 1.7.

Proof of Theorem 1.3. Under our weak condition (C3)', Lemma 2.1 is also true by replacing $a_{i j}(x)$ with $\delta_{i j}$. In fact in the proof of Lemma 2. 1 the term related to $q_{1}(x)$ is

$$
\begin{gathered}
\int_{B(s, t)}\left(2 f q_{1} \operatorname{Re}[\langle\hat{x}, \overline{D v}\rangle v]+g q_{1}|v|^{2}\right) d x \\
=\int_{B(s, t)}\left(f q_{1} \partial_{r}\left(|v|^{2}\right)+g q_{1}|v|^{2}\right) d x,
\end{gathered}
$$

which, noting Lemma 4.1 , can be integrated by parts. Then Lemma 2.2 and the proof of Theorem 1.1 is also true, if we replace $a_{i j}(x)$ with $\delta_{i j}$.

Proof of Theorem 1.4. Noting Theorem 1.3 we have the assertion by the same way as the proof of Theorem 1.2.

Proof of Theorem 1.5. For any $\varepsilon>0$ let

$$
\mu= \begin{cases}(1+\varepsilon) \sqrt{|\lambda|,} & \text { if } \lambda<0, \\ \varepsilon / 2, & \text { if } \lambda \geq 0 .\end{cases}
$$

Then this $\mu>0$ satisfies (\#\#). So by Theorem 1.4 we have the assertion.

Proof of Theorem 1. 6. Replacing $a_{i j}(x)$ with $\delta_{i j}$ and $b_{i}(x)$ with 0 , Lemma 2.1 is also true. Then we have

$$
\begin{aligned}
& F(t ; \rho, f, g)-F(s ; \rho, f, g) \\
& \quad=\int_{B(s, t)}\left[2\left(2 \rho^{\prime} f+f^{\prime}-r^{-1} f\right)\left|\partial_{r} v\right|^{2}+\left(2 r^{-1} f+g-f \operatorname{div}(\hat{x})\right.\right. \\
& \left.\quad-f^{\prime}\right)|\operatorname{grad} v|^{2}+2 \operatorname{Re}\left[\left(f\left(q_{2}+k_{2}\right)+g \rho^{\prime}+2^{-1} \partial_{r} g\right)\left(\overline{\left.\partial_{r} v\right)} v\right]\right. \\
& \quad+\operatorname{Re}\left[\left\langle\left(\operatorname{grad} g-\hat{x} \partial_{r} g\right), \overline{\operatorname{grad} v\rangle}\right]+\left\{\left(q_{1}-\lambda+k_{1}\right)\left(g-f \operatorname{div}(\hat{x})-f^{\prime}\right)\right.\right. \\
& \left.\left.\quad-f \partial_{r}\left(q_{1}+k_{1}\right)+g\left(\operatorname{Re}\left[q_{2}\right]+k_{2}\right)\right\}|v|^{2}\right] d x .
\end{aligned}
$$

In the above let

$$
\begin{aligned}
& f(r)=1, g(x)=f \operatorname{div}(\hat{x})+f^{\prime}-\gamma(x) r^{-1}=(n-1-\gamma(x)) r^{-1}, \\
& \rho(r)=\mu r+m r^{l} .
\end{aligned}
$$


Then we have

$$
\begin{aligned}
& \left(2 r^{-1} f+g-f \operatorname{div}(\hat{x})-f^{\prime}\right)|\operatorname{grad} v|^{2} \\
& \quad+\operatorname{Re}\left[\left\langle\left(\operatorname{grad} g-\hat{x} \partial_{r} g\right), \overline{\operatorname{grad} v}\right\rangle\right] \\
& \geq r^{-1}\left(2-\gamma(x)-\left|\operatorname{grad} \gamma(x)-\hat{x} \partial_{r} \gamma(x)\right|^{\alpha}\right)|\operatorname{grad} v|^{2} \\
& \quad \quad-(4 r)^{-1}\left|\operatorname{grad} \gamma(x)-\hat{x} \partial_{r} \gamma(x)\right|^{2-\alpha}|v|^{2} \\
& \geq-r^{-1} \varepsilon_{15}(r)|v|^{2} .
\end{aligned}
$$

So replacing $a_{i j}(x)$ with $\delta_{i j}$ and $b_{i}(x)$ with 0 , Lemma 2.2 and the proof of Theorem 1.1 are also true under our weak conditions (C3)' and (C4)'。

Proof of Theorem 1. 7. By the same way as the proof of Theorem 1.2 we have the assertion.

\section{References}

[1] Agmon, S., Lectures on exponential decay of solutions of second-order elliptic equations: bounds on eigenfunctions of $N$-body Schrödinger operators, Mathematical Notes 29, Princeton University Press and University of Tokyo Press, 1982.

[2] Bardos, C. and M. Merigot, Asymptotic decay of the solution of a second-order elliptic equation in an unbounded domain. Applications to the spectral properties of a Hamiltonian, Proc. Roy. Soc. Edinburgh, $76 \mathbb{A}$ (1977), 323-344.

[3] Eastham, M. S. P. and H. Kalf, Schrödinger-type operators with continuous spectra, Research notes in mathematics 65, Pitman Advanced Publishing Program, 1982.

[4] Éidus, D. M., The principle of limit amplitude, Usp. Math. nauk, 24 (1969), Vypusk 3 (147), 91-156.

[5] Ikebe, T. and J. Uchiyama, On the asymptotic behavior of eigenfunctions of secondorder elliptic operators, J. Math. Kyoto Univ., 11-3 (1971), 425-448.

[6] Kalf, H., Non-existence of eigenvalues of Schrödinger operators, Proc. Roy. Edinburgh, 79 A (1977), 152-172.

[7] Mochizuki, K., Growth properties of solutions of second order elliptic differential equations, J. Math. Kyoto Univ., 16-2 (1976), 351-373.

[8] Mochizuki, K. and J. Uchiyama, On eigenvalues in the continuum of 2-body or many-body Schrödinger operators, Nagoya Math. J., 70 (1978), 125-141.

[9] Roze, S. N., On the spectrum of a second-order elliptic operator, Math. Sb., 80 (112) (1969), 195-209 (Russian).

[10] Uchiyama, J., Lower bounds of growth order of solutions of Schrödinger equations with homogeneous potentials, Publ. RIMS, Kyoto Univ., 10 (1975), 425-444. 
